\title{
El ámbito de la libertad individual en la teoría fichteana del derecho natural de 1796/1797
}

Héctor Arrese Igor

\section{(2) OpenEdition \\ Journals}

Edición electrónica

URL: http://journals.openedition.org/ref/342

ISSN: 2258-014X

Editor

EuroPhilosophie Editions

\section{Referencia electrónica}

Héctor Arrese Igor, «El ámbito de la libertad individual en la teoría fichteana del derecho natural de 1796/1797 », Revista de Estud(i)os sobre Fichte [En línea], 2 | 2011, Publicado el 22 noviembre 2011, consultado el 08 septiembre 2020. URL : http://journals.openedition.org/ref/342

Este documento fue generado automáticamente el 8 septiembre 2020.

(c) EuroPhilosophie 


\title{
El ámbito de la libertad individual en la teoría fichteana del derecho natural de 1796/1797
}

\author{
Héctor Arrese Igor
}

1 Johann G. Fichte defendió con firmeza las libertades individuales, en particular la libertad de expresión, ya desde sus primeros escritos en torno a la Revolución Francesa1. Allí Fichte desarrolló su convicción de que "el hombre no puede ser heredado, vendido o regalado." (ZDF, GA I/1, 173/FSW, VI, 11) En especial sostuvo que la libertad de expresión es indispensable en una sociedad racional, porque el objetivo de todo Estado es garantizar las condiciones para el desarrollo de la moralidad de sus ciudadanos. (cf. ZDF, GA I/1, 176-177/FSW, VI, 15.) Esto significa que, si los ciudadanos no pueden intercambiar las razones que tienen para sostener sus convicciones morales, no podrán tampoco progresar en el camino de la autonomía moral, es decir en el proceso de darse a sí mismos las normas para su acción. (cf. ZDF, GA I/1, 174-178 y 183/ FSW, VI, 13-17 y 24) No puede objetarse a este reclamo que aquellas convicciones morales, que son diferentes respecto de las que uno sostiene, deberían ser prohibidas porque lesionan la propia sensibilidad, porque nadie está obligado a prestar atención a aquellas opiniones que considera nocivas para su integridad moral. (ZDF, GA, I, 1, 176-177 / FSW, VI, 15)

2 Los ciudadanos suscribirían el contrato social, en el marco de la teoría fichteana de 1793/1794, para proteger su derecho inalienable a la autonomía moral, por medio de la cesión mutua de su derecho alienable a las condiciones exteriores de la moralidad: las libertades de expresión y reunión, los derechos de propiedad, el derecho a hacer uso de determinados privilegios y prerrogativas, etc.. (ZDF, GA I/1, 174-175 / FSW, VI, 13.) De este modo, el contrato podrá ser anulado, si el ciudadano considera que el soberano no ha garantizado sus derechos básicos².

3 Ahora bien, en el Grundlage des Naturrechts (en adelante GNR), publicado en 1796/1797, Fichte pareciera colocar a la cuestión de las libertades individuales en un segundo plano, porque el Estado tiene como una función prioritaria la garantía de los derechos 
de propiedad de cada ciudadano, sobre todo el derecho a poder vivir del propio trabajo. Para lograr este objetivo, Fichte propone la concentración de los poderes ejecutivo, legislativo y judicial en el soberano, cuya acción gubernamental podrá ser controlada solamente por el órgano del eforato, que deberá denunciar cualquier proceder que sea inconstitucional. (GNR § 16, GA I/3, 432-460 / FSW, III, 150-187) De este modo, Fichte coloca una barrera que parece insalvable entre los ciudadanos particulares de carne y hueso y el poder político, sólo mediada por el eforato, que deberá convocar a una asamblea popular para juzgar al gobierno en caso de tener pruebas evidentes en su contra. Entonces la soberanía popular queda restringida sólo a los casos excepcionales de la implementación de un juicio político contra las autoridades ${ }^{3}$.

El individuo quedaría entonces al margen del funcionamiento cotidiano del Estado, no solamente a causa del régimen político que Fichte propone, sino también en virtud de una razón adicional. El ciudadano también estaría preso de la mecánica de la planificación central de la economía. El Estado planifica centralmente la división social del trabajo y la actividad del comercio, a fin de evitar que la libertad y la propiedad de los ciudadanos quede a merced del azar y la arbitrariedad del mercado libre. Cada ciudadano deberá entonces producir la cantidad de mercancías que el Estado le ordene, luego entregarlas a los almacenes públicos y consumir sólo los bienes que el Estado considera que le son indispensables para la vida. Esta idea de una economía planificada centralmente por el Estado para subvenir a las necesidades de sus ciudadanos ejerció una influencia considerable en el socialismo alemán decimonónico ${ }^{4}$.

5 Sin embargo, considero que la teoría fichteana del derecho natural protege y garantiza también las mismas libertades individuales que se defendían en los escritos de 1793/1794. En primer lugar, el derecho originario, que está a la base de la Constitución misma del Estado, consiste en la capacidad de desencadenar cursos libres de acción en el mundo sensible, lo que significa ante todo la garantía del derecho de propiedad, objeto de la planificación central de la economía. Es decir que el pensamiento de Fichte no puede ser interpretado como una forma de colectivismo, porque gira en torno a la inviolabilidad del derecho que tiene cada individuo de actuar como mejor le parezca.

6 Algunos intérpretes de Fichte han puesto de relieve este costado de su teoría del Estado. A. Renaut argumenta que en la teoría de Fichte conviven, junto con el momento hobbesiano, también otros dos, integrantes todos de lo que Renaut denomina como la síntesis republicana del GNR. El primero sería un momento rousseauniano, según el cual el pueblo es el poder supremo y origen de todo poder. El segundo sería de tipo lockeano, y consistiría en una crítica de la democracia directa como una forma de despotismo y en la defensa de la democracia representativa. Finalmente, esta síntesis estaría integrada por un momento hobbesiano, marcado por la unidad de los poderes legislativo, ejecutivo y judicial. (cf. GNR, GA I/3, 450-452/FSW III, 174-175) 5 De este modo, Renaut sostiene que el modelo fichteano de Estado tiene una complejidad tal que no puede ser pensado como un régimen despótico.

7 N. Nomer ha refutado las interpretaciones colectivistas del pensamiento de Fichte, mostrando que la teoría fichteana del derecho natural tiene por objeto garantizar el libre desarrollo de la individualidad de cada persona ${ }^{6}$. Como bien señala Nomer, los ciudadanos del Estado fichteano tienen la obligación de respetar al poder ejecutivo sólo si garantiza su derecho originario a las libertades, prerrogativas y bienes que les permiten iniciar cursos de acción en el mundo sensible. Esta es la función que los ciudadanos le han encomendado al Estado, y el motivo por el que han decidido entrar 
en el estado civil y suscribir el contrato social. Nomer explica el sentido profundo que tiene la idea fichteana de una economía planificada centralmente por el Estado, que no es otro que distribuir la propiedad de modo tal que cada uno pueda vivir del trabajo o del oficio que ha elegido libremente ${ }^{7}$.

Pero el espacio que Fichte deja a las libertades individuales está determinado por la distribución de aquellos bienes que denomina como de propiedad absoluta, que consiste fundamentalmente en el uso privado del dinero, pero que también tiene que ver con el derecho a dejar en herencia los propios bienes luego de la muerte $\mathrm{y}$, finalmente, con el derecho a la privacidad en el ámbito doméstico. En este trabajo intentaré reconstruir la argumentación que Fichte esgrime en su teoría del derecho de 1796/1797 para delimitar este espacio de la individualidad libre. De este modo es mi intención alejar toda sospecha de colectivismo y mostrar el delicado equilibrio que busca Fichte entre la individualidad de cada ciudadano y la comunidad estatal.

\section{La teoría del dinero en el derecho natural de 1796/97}

La teoría del dinero es parte integrante de la concepción fichteana de la división del trabajo, porque es el medio que le compete por excelencia a la clase de los comerciantes. Ellos son los responsables de hacer circular la moneda, porque son los encargados de devolver a los ciudadanos el sobrante de sus impuestos en forma de dinero. Para comprender la argumentación de Fichte es necesario, en primer lugar, considerar los contratos de ciudadanía, que incluyen al contrato de propiedad y al de protección. Según el contrato de propiedad, cada ciudadano conserva un espacio de libertad inalienable y se abstiene de interferir en el de los demás pero, en virtud del contrato de protección, se compromete a ceder parte de su libertad para proteger la libertad de los otros ${ }^{8}$.

Entonces pareciera que la libertad protegida por el contrato de propiedad es inalienable, pero a la vez puede ser alienada para proteger a los demás ciudadanos. Podría decirse que la libertad inalienable del sujeto es el espacio de acción que queda luego de la cesión de su libertad en el contrato de protección. Pero nunca puede resultar del contrato de protección una cantidad insignificante de libertad, porque entonces el contrato mismo perdería sentido, toda vez que no habría una cantidad razonable de libertad que proteger.

11 Es importante, a fin de comprender la argumentación que sigue, tener presente que la libertad significa en este contexto la distribución de la propiedad. Fichte intenta resolver, por medio de la teoría del dinero, esta tensión entre la libertad que necesita el Estado, a fin de poder proteger a los ciudadanos, y la libertad de los ciudadanos, que es aquello que debe ser protegido.

12 Aun cuando los ciudadanos necesiten de una cantidad determinada de propiedad para poder realizar sus fines individuales, según el derecho originario, el Estado no podrá garantizarles tal o cual bien particular, como sucede en el caso de la protección del derecho a la existencia. El derecho a la existencia es una parte insustituible del derecho originario, porque garantiza la posibilidad misma de que el sujeto pueda actuar en el mundo sensible por medio de su cuerpo articulado. El derecho a la existencia puede ser garantizado sólo si se distribuye entre los ciudadanos lo necesario para su alimentación, vivienda, abrigo, etc., y se garantiza su acceso a la salud. Es decir, las condiciones del derecho a la existencia son las mismas para todos los ciudadanos, 
porque se trata de las mismas necesidades básicas, si bien deben ser satisfechas de acuerdo con la ocupación que desempeñe cada ciudadano. Por esta razón, el Estado puede planificar centralmente la aplicación del derecho a la existencia. Pero la cantidad indeterminada de fines que el sujeto se propone a título individual no puede ser planificada del mismo modo, porque requiere de una cantidad y variedad infinita de objetos, en calidad de medios.

Fichte propone una instancia mediadora entre los bienes que se poseen y la satisfacción de fines indeterminados, que no es otra que el dinero. El dinero debe garantizar esta propiedad de carácter indeterminado, porque representa la cantidad de propiedad que el ciudadano ha producido. Pero debe posibilitarle al ciudadano al mismo tiempo la adquisición de una diversidad de bienes, atendiendo a los múltiples fines de sus acciones. Por esta razón el dinero tiene un carácter simbólico, esto es, representa una cantidad determinada de propiedad.

El dinero debe estar constituido de modo tal que lo material en él sea insignificante en orden a la satisfacción de fines específicos. En caso contrario, perdería su carácter simbólico. Para aclarar esto con un ejemplo del mismo Fichte, no es conveniente que el dinero esté acuñado con plata, dado que este metal mismo puede ser trabajado en orden a la producción de bienes determinados (utensilios, etc.), que tienen un valor en sí mismos. (GNR § 19, GA I/4, 42-43/FSW III, 239) Si el dinero tuviera un valor en sí mismo, entonces ya no cumpliría con su función de representar el valor de otra cosa. Por eso Fichte propone fabricar el dinero con cuero o papel ${ }^{9}$.

Ahora bien, no sólo es importante el material del que está hecho el dinero, sino también el modo de su circulación. El ciudadano entrega todo el producto de su trabajo al Estado, puesto que éste es el encargado de distruibuir la propiedad entre los ciudadanos $\mathrm{y}$, en última instancia, de velar por el cumplimiento del derecho a la propiedad. El Estado calcula, en base a la cantidad de productos que ha recibido, el valor total de los bienes de que se dispone y, de este modo, fija el valor del dinero. Independientemente de la cantidad de moneda que se emita o circule, el valor del dinero permanecerá siempre vinculado a la suma total de bienes producidos ${ }^{10}$.

16 Si la cantidad de dinero circulante es mayor que la suma total de bienes, su valor decrecerá $\mathrm{y}$, en caso contrario, se incrementará. Luego de haber recibido todos los productos de manos de los ciudadanos, el Estado debe redistruibuirlos, en primer lugar, en razón de las necesidades vitales de los contribuyentes. Una vez garantizado el derecho a la existencia, el Estado debe calcular cuántos de estos productos necesita para proveer al cumplimiento de sus funciones básicas, en el espíritu del contrato de protección.

17 El sobrante es devuelto a los ciudadanos en proporción a la cantidad que han producido. Dado que los ciudadanos ya tienen garantizado su derecho a la existencia, necesitan del resto del producto de su trabajo para realizar sus fines particulares. Por lo tanto, debe devolverse al ciudadano lo que le corresponde en forma de dinero, para que haga de esta propiedad el uso que quiera. El ciudadano que posee dinero, entonces, ya ha honrado previamente sus obligaciones impositivas.

18 La disposición de dinero es el mejor certificado del cumplimento de los contratos de ciudadanía. Fichte rechaza la implementación de un impuesto al dinero porque el dinero es, por definición, el sobrante que queda luego de que se han pagado todos los impuestos. Fichte entiende que su teoría minimiza los riesgos de sobreemisión de 
moneda, porque el valor del dinero es estable, toda vez que es calculado en base a la cantidad de mercancías disponible.

\section{La propiedad del dinero}

19 Fichte establece el derecho de cada ciudadano a disponer de una vivienda inviolable a partir de un problema práctico que ha surgido de la teoría del dinero. El punto de partida de Fichte consiste en que el Estado debe garantizar a cada ciudadano su propiedad, entregada en forma de dinero luego de que haya pagado sus impuestos. Se trata de una propiedad absoluta o inviolable y que es un objeto de protección del Estado, según el contrato de ciudadanía. Pero esta conclusión deja abierto un problema práctico, porque no queda claro cómo puede el Estado garantizar la propiedad del dinero, dado que no se puede establecer un signo unívoco que la indique.

Por el contrario, la propiedad del suelo es indicada por el signo unívoco de los mojones. De este modo pueden resolverse con facilidad y de modo expeditivo los litigios relativos a la propiedad del suelo cultivable. Ahora bien, en el caso del dinero, el Estado no puede disponer de ningún signo que indique que estos billetes pertenecen a tal ciudadano y no a otro. Dicho de otra manera, la naturaleza misma del dinero es tal que circula de modo anónimo de una mano a otra, y que no presenta marca de propiedad particular alguna ${ }^{11}$.

21 Pero la propiedad del dinero presenta otro problema, dado que aún en el caso de que el Estado pudiera determinar quién es el propietario de tal o cual billete, no estaría legitimado para adquirir este conocimiento, porque se trata de una propiedad absoluta. Lo que cada ciudadano hace con su dinero pertenece a su esfera privada, que debe ser protegida por la ley. Esto la sustrae claramente al control del Estado porque, si fuera controlada, el sujeto no podría someter el dinero al uso absolutamente espontáneo y libre de su voluntad, ya que debería subordinarse enteramente a las reglas del Estado. (GNR § 19, GA I/4, 44/FSW III, 241)

22 Fichte resuelve este problema con dos estrategias argumentativas. Pero ambas tienen algo en común: se trata de encontrar un elemento que la persona pueda controlar de modo inmediato y que esté vinculado directamente al dinero. Pero este elemento determinado y determinable por la persona debería dejar el uso del dinero librado a su exclusiva privacidad. De este modo, cada ciudadano podría indicar ostensiblemente ante los demás, si quisieran apoderarse de su dinero, que es de su propiedad y, sin embargo, conservar el derecho de utilizarlo como quiera.

La primera estrategia consiste en indicar un elemento particular con el que la persona está inmediatamente vinculada. No hay otro elemento vinculado más inmediatamente con la persona que su cuerpo, según lo argumentado en los $\S \S 5$ al 7 del GNR. Esto es, si la persona lleva en los vestidos que están sobre su cuerpo una cierta cantidad de dinero, y no media denuncia de robo contra ella, ésto será siempre un signo incontestable de su propiedad, en el caso de que otra persona pretendiera reclamarlo como propio. (GNR, § 19; GA, I, 4, 44-45 / FSW, III, 242.)

La segunda estrategia demuestra la necesidad de que el ciudadano disponga de una vivienda privada. Esto se desprende a partir del uso que se espera que el ciudadano haga de su dinero. Cada ciudadano no utiliza su dinero sólo para satisfacer sus necesidades presentes, sino que es de esperar que también guarde una parte para 
utilizar en vista de sus necesidades futuras. La razón de esto es que se prevé que el ciudadano utilice su dinero en el período que va desde el pago de los impuestos al plazo siguiente de pago. Se supone que cada ciudadano tendrá necesidades que satisfacer a lo largo de todo este período y no sólo al comienzo.

Pero no puede esperarse que el ciudadano lleve todo el tiempo la suma completa de dinero en sus vestidos sobre su cuerpo, porque no sólo sería algo poco práctico, sino sobre todo inseguro respecto de los ataques de los demás ${ }^{12}$. Puede disponerse, sin embargo, de una solución análoga a la que ya hemos visto. Pero el dinero no puede vincularse ya más de modo inmediato con la persona, sino por medio de otra cosa. Se trata de la vivienda, figura que en Fichte significa no sólo la propiedad de una casa o el alquiler de una habitación, sino también todo aquel espacio en el cual el ciudadano puede poner a seguro su dinero. Ejemplos de esto son el baúl de la sirvienta (die Lade der Dienstmagd), el cofre que uno entrega al correo (der Koffer, der auf die Post gegeben wird), etc. La cerradura de la propia vivienda marca el límite de nuestra privacidad, y todo el que irrumpa allí violentamente y sin nuestro consentimiento debe ser castigado por el Estado"13: "El control del Estado se extiende hasta la cerradura de la puerta, y a partir de allí comienza el mío. La cerradura es la frontera que separa el poder del Estado del poder privado. "(GNR § 19, GA I/4, 44-45/FSW III, 242).

De este modo, el derecho a una vivienda se integra de modo indisoluble al conjunto de los derechos de ciudadanía.

\section{El problema del manejo privado del dinero}

El ciudadano puede utilizar del modo en que quiera su propiedad absoluta, que recibe del Estado en forma de dinero. Pero las transacciones que el ciudadano lleva a cabo en su esfera privada tienen consecuencias para su propiedad, por lo que pareciera ser que el Estado tiene el derecho y la obligación de controlarlas y regularlas. De este modo, el Estado garantizaría al ciudadano una esfera de propiedad inviolable, y se reservaría a la vez el derecho de intervenir en esta esfera, lo que es contradictorio.

Fichte desarrolla diversas estrategias para que el Estado evite o prevenga que los ciudadanos sean perjudicados a consecuencia de sus transacciones privadas, que contemplan desde los casos de estafas hasta la donación y el testamento. No se puede permitir que el Estado pueda de alguna manera violar la propiedad absoluta de los ciudadanos, porque de este modo se transgrediría el contrato de propiedad, que es un momento fundamental del contrato de ciudadanía ${ }^{14}$. Por lo tanto, todas las transacciones que los ciudadanos quieran realizar en el ámbito informal de sus relaciones privadas quedan libradas a su leal saber y entender y no son asunto del Estado.

El Estado no podría evitar las estafas, porque no cuenta, entre sus atribuciones, con la potestad de obligar en lo concerniente a la buena fe o la confianza entre los ciudadanos. La propiedad de los ciudadanos sería necesariamente insegura, porque ellos tienen derecho a prestar dinero de buena fe a otros sin el control del Estado, esto es, sin recurrir a pagarés o letras de cambio. El Estado mismo es fruto de la desconfianza entre los hombres, por lo que no puede él mismo obligarlos a que confíen mutuamente unos en otros. Pero esto tendría además consecuencias indeseables desde el punto de vista legal ya que, si el Estado coaccionara a los ciudadanos para que confíen mutuamente en los otros, al mismo tiempo los obligaría a poner en riesgo su propiedad, mientras que su 
obligación primordial es resguardarla y custodiarla. Por otro lado, la buena fe y la confianza mutua caen en el ámbito de la moralidad, es decir, fuera de la jurisdicción del derecho y, por lo tanto, del Estado.

El estafador que ha abusado de la confianza y la buena fe de los otros ciudadanos, sin embargo, les ha producido un daño considerable y queda a consecuencia de ello deshonrado ${ }^{15}$. Dicho de otra manera, los demás ciudadanos ya no confiarán en la fidelidad del estafador a su palabra dada. Pero puede ocurrir que otros ciudadanos que no conocen la estafa sean engañados en el futuro, por lo cual el Estado puede hacerlo público y deshonrar al estafador ante todos. Esta es una alternativa razonable, porque no implica ningún control ni regulación de las transacciones privadas.

El ciudadano deshonrado ha recibido suficiente castigo con la pérdida de la confianza de los demás, por las consecuencias que esto acarrea para sus relaciones sociales. El honor es un bien muy estimado por los hombres por la relevancia que tiene para desarrollar sus vínculos entre sí. Por eso el ciudadano que ha sido deshonrado sin causa alguna, esto es, que ha sido injuriado o difamado, tiene el derecho, en sí meramente negativo, de exigir al Estado que le restituya su honor y se retracte en público.

La donación y el testamento son casos de adquisición de la propiedad que presentan una cierta complejidad, porque en ellos no se trata de la mera adquisición original de la propiedad por parte del Estado ni del comercio usual entre los ciudadanos. En el caso de la adquisición original de la propiedad, el Estado acrecienta su patrimonio en virtud de la cesión de la propiedad de los ciudadanos, de acuerdo con el contrato de protección. En cambio, las relaciones comerciales implican la cesión de cada ciudadano de su propiedad a otro a cambio de otra de valor similar, pero esto no implica un incremento de la propiedad total, sino que sólo hace referencia a su circulación.

$\mathrm{Ni}$ la adquisición original ni el comercio presentan problemas teóricos especiales, porque quedan incluidos bajo la órbita del contralor del Estado. Fichte pone dos ejemplos que ilustran claramente cómo debe controlar el Estado las transacciones comerciales entre los ciudadanos. (GNR § 19, GA I/4, 55-56/FSW III, 256) En primer lugar, el Estado debe cuidar de que la mercadería adquirida por medio del comercio sirva a la profesión o al oficio que desempeña el ciudadano que la ha comprado. En caso contrario, el ciudadano abandonaría su propiedad para conseguir otra, de cuyo uso no se beneficiaría el Estado. Es decir, del uso de esta propiedad no se seguiría el fortalecimiento del Estado, por lo cual se desperdiciaría un recurso necesario para la protección de la propiedad de todos. En segundo lugar, el Estado tiene la obligación de controlar que ningún ciudadano enajene toda su propiedad en sus transacciones comerciales, sino que siempre conserve un resto para procurar su subsistencia. La razón de esto es que, de acuerdo con el contrato de ciudadanía, el Estado es responsable de la autoconservación de todos.

Por lo tanto, el Estado puede intervenir en las transacciones comerciales que tienen lugar en el ámbito público. Pero en la donación y el testamento ocurre algo muy diferente porque, por medio de estos procesos, un ciudadano transfiere su propiedad a otro, total o parcialmente, sin recibir nada a cambio. Ambas transacciones pertenecen al derecho doméstico, lo que dificulta su regulación estatal. Es decir, en los dos casos el ciudadano intercambia con los demás su propiedad absoluta, de la que dispone con total independencia. propiedad es legal en tanto que es reconocida estatalmente, pero a la vez estas 
transacciones tienen lugar en el ámbito privado, que en principio al menos escapa al control del Estado. Esta es la tensión que Fichte busca resolver con su teoría de la donación y el testamento. En el caso de la donación, sin embargo, el Estado tiene claramente la potestad de intervenir, aunque se trate del derecho doméstico. La razón de esto es que la donación puede poner en peligro la autoconservación misma del ciudadano, por lo cual se le aplica el requisito antes mencionado para las transacciones comerciales. Dicho de otra manera: la donación de una propiedad puede traer como consecuencia tanto que el donante enajene un recurso que necesita para su trabajo, como que entregue aquello que necesita para vivir. Por eso la donación debe ser declarada ante el Estado, para que pueda efectuarse la fiscalización correspondiente. Si el ciudadano que cede parte de su propiedad conserva lo necesario para ejercer su profesión o para seguir viviendo, entonces puede tener lugar la donación y ser reconocida por el Estado.

El testamento, por otro lado, no exige ni permite controles como los propuestos para la donación, porque no se trata de la transferencia de un bien por parte de un ciudadano que permanece existiendo, sino que tendrá lugar sólo luego de su muerte. Por lo tanto, aquí no se presenta el problema de si el ciudadano está enajenando con este acto algo necesario para realizar su oficio o para su autoconservación. Pero, como resultado del testamento, un ciudadano o un grupo de ellos incrementa su propiedad, lo cual de por sí reclama el control del Estado, porque toda propiedad puede ser reconocida sólo por su intermedio.

Podría sostenerse simplemente que, una vez muerto el propietario, sus bienes pasarían automáticamente a ser propiedad del Estado, en virtud del contrato de protección. El contrato de protección entraría en tensión, de este modo, con el contrato de propiedad, según el cual cada individuo tiene derecho a disponer libremente de su propiedad absoluta. Sin embargo, no se da en realidad esta tensión en la figura del testamento, porque el ciudadano no asegura aquí su propiedad, toda vez que ya no existirá más luego de completada la transferencia, ni la propiedad del Estado, porque este último tiene derecho a exigir su contribución al ciudadano mientras permanezca existiendo.

Por otro lado, puede invocarse un motivo suficiente para exigir la regulación del testamento. La herencia es un bien para los ciudadanos, ya que tiene consecuencias importantes para su vida. Baste para ello pensar en los efectos que tiene para el propietario la convicción de sus herederos de que recibirán sus bienes luego de su muerte. Esta convicción puede motivar a los herederos a cuidar mejor del ciudadano en vida, a expresarle su afecto, etc. (cf. GNR § 19, GA I/4, 56-58/FSW III, 257-258) Los ciudadanos tienen derecho a pedir al Estado que legisle en todos los aspectos de su vida en los que consideren necesaria una regulación especial. Por ello es necesario que el Estado garantice que los bienes dejados en herencia serán recibidos por los herederos, según la voluntad del difunto. En consecuencia, el Estado debe comprometerse a reconocer la propiedad heredada ${ }^{16}$.

Los herederos se ocuparán con seguridad de que se sigan todos los procedimientos establecidos por la ley, porque está en juego su adquisición de la herencia. Sin embargo, la regulación del derecho sucesorio no es sencilla porque el Estado, en caso de que la herencia no corresponda a quienes la reclaman, será el beneficiario directo de esta propiedad. Por lo tanto, si el Estado mismo fuera responsable del proceso de la sucesión, sería juez y parte en el mismo asunto, lo cual es contradictorio. 
40 En consecuencia, en el proceso sucesorio deben presentarse dos partes: los herederos, por un lado, y el Estado, por el otro. De este modo, debe preverse por ley la actuación de un funcionario que no esté bajo el control del Estado, sino de la única comunidad que resta, es decir el pueblo. Entonces el magistrado que instruya el proceso de la sucesión debe poder ser controlado y denunciado por cualquier ciudadano. El pueblo se constituye como representante en la persona de los testigos (Zeugen), quienes tienen la función de controlar que la herencia cumpla con todas las condiciones previstas por la ley.

\section{Conclusiones}

41 El punto de partida de la teoría fichteana del derecho natural es la deducción de las condiciones que son necesarias para la autoconciencia del yo, que a su vez consiste en la atribución del yo a sí mismo de la capacidad de conocer objetos en el mundo sensible. Como resultado de su argumentación, Fichte afirma que la autoconciencia del yo es posible sólo gracias a la acción de otro yo, que lo reconozca como un ser racional y, por lo tanto, como alguien capaz de realizar acciones libres en el mundo sensible. Ahora bien, el otro puede reconocer al yo sólo por medio de una acción en la que no lo coaccione para realizar tal o cual acción particular, sino que le deje abierto un conjunto de alternativas de acción. Este es justamente el sentido del principio del derecho (Rechtssatz), según el cual cada sujeto deberá delimitar su esfera de acción, dejando otra esfera igual para los demás. Es decir que el derecho es la condición de posibilidad de la autoconciencia del Yo.

Las teorías fichteanas del Estado y de la división social del trabajo consisten en la aplicación del principio del derecho. Como se dijo anteriormente, el Estado fichteano unifica los poderes ejecutivo, legislativo y judicial en las manos del gobernante, para facilitar la aplicación eficiente del principio del derecho por medio de la ley. Pero la aplicación del principio del derecho requiere a su vez de la planificación centralizada de la economía, para que cada ciudadano reciba la cantidad de propiedad que necesita para poder vivir de su trabajo. El contrato mismo de ciudadanía exige que cada uno declare ante el Estado el oficio que quiere ejercer y que reciba su aprobación, que deberá venir seguida de la garantía de los medios para realizarla. De este modo, el ciudadano se verá obligado a cumplir con las exigencias que le plantee el Estado, de acuerdo con el rol que juegue en la planificación centralizada de la economía.

Ahora bien, pareciera entonces que Fichte se ha ido desplazando desde la necesidad de garantizar que cada yo individual acceda a la autoconciencia, gracias a la disposición de una esfera adecuada de acciones, hasta la idea de un Estado despótico donde el poder esté concentrado en pocas manos y cada ciudadano se convierta en un mero engranaje de una maquinaria implacable que coordine la economía de la comunidad. Esto significa que Fichte habría abandonado la prioridad absoluta que tiene el individuo en su teoría de la autoconciencia, a favor de la idea de un Estado dominante y autoritario.

Sin embargo, la teoría fichteana del dinero pone en evidencia que su idea de la planificación centralizada de la economía tiene como función la transferencia de recursos a los ciudadanos para que puedan realizar sus preferencias personales. Se trata de la idea de la propiedad absoluta, sobre cuyo uso el Estado no puede tener injerencia alguna, salvo que este uso atente contra la autoconservación del ciudadano o contra el ejercicio de su oficio. Lo mismo ocurre con la figura legal de la donación. Por 
el contrario, el testamento por el cual un ciudadano deja sus bienes en herencia constituye una esfera de la individualidad que debe ser respetada, del mismo modo que la vida que el ciudadano lleve dentro de su casa.

De este modo, puede concluirse que el Estado fichteano del GNR no es una forma de colectivismo que asfixie a la individualidad de cada ciudadano, pero que tampoco deja a cada uno librado a su suerte, sino que busca un equilibrio complejo entre un sistema de producción distribución centralizada de las mercancías, por un lado, y un individuo que pueda realizar sus preferencias particulares, por el otro.

\section{NOTAS}

1. Me refiero al texto Zurückforderung der Denkfreiheit (publicado en 1793, en adelante ZDF) y a Beitrag zur Berichtigung der Urtheile des Publicums über die französische Revolution (1793).

2. G. RAMETTA, "Politik der Vernunft und Vernunftstaat bei Fichte (1793-1808)“, en: C. DE PASCALE et al., (eds.), Fichte und die Aufklärung, Hildesheim-Zürich-New York: G. Olms, 2004, pp. 227-241. Aquí: p. 230.

3. F. ONCINA COVES, "Fichtes Kritik des aufklärerischen Republikanismus", en: C. DE PASCALE et al., (eds.), Fichte und die Aufklärung, pp. 213-226. Aquí: 224-225.

4. Cfr. A. DAMIANI, "El Fichte de Lasalle: antecedentes e influencias“, en: Deus Mortalis, № 7, 2008, pp. 253-275.

5. A. RenAUT, Le Système de Droit. Philosophie et droit dans la pensée de Fichte, Paris: PUF, 1986 , pp. $378-379$

6. N. NOMER, "Fichte and the Idea of Liberal Socialism", in: The Journal of Political Philosophy, Volume 13, Number 1, 2005, pp. 53-73. Aquí p. 73.

7. N. NOMER, "Fichte and the Idea of Liberal Socialism", pp. 60-70.

8. Acerca del contrato de propiedad véase GNR $\S 17, \mathrm{GA} I / 4,8 / \mathrm{FSW}$ III, 195. Respecto del contrato de protección véase GNR $\$ 17$, GA I/4, 9-10/FSW III, 197.

9. Para el comercio exterior, Fichte prefiere que el dinero sea fabricado con plata y oro, porque son metales que conservan un valor similar entre los Estados, y evitaría los problemas que acarrea la conversión de la moneda de cada país en la del otro. Esta es una razón adicional para que el Estado monopolice la explotación de la minería. (cf. GNR § 19, FSW III, 239)

10. Sólo el Estado puede acuñar dinero, porque sólo él está en condiciones de garantizar su valor. (cf. GNR § 19, GA I/4, 42-43/FSW III, 239)

11. "Que el campo que se sitúa entre tal y tal trozo de tierra, designado por tales mojones, me pertenece y no pertenece a ningún otro, esto debe estar puesto por escrito en los registros de mi localidad; y si a este respecto surge un litigio, los mismos registros decidirán sin necesidad de otra cosa. Pero, ¿cómo puede indicarse que este tálero determinado me pertenece y no pertenece a ningún otro? Todos los táleros se 
parecen y deben parecerse porque están destinados a cambiar de propietario sin otra formalidad." (GNR § 19, GA I/4, 43-44/FDN, 299)

12. Como señala Fichte, los vagabundos (Lazzaronis) llevan todo el dinero en sus vestidos, pero esto es justamente un signo de su desprotección. (GNR § 19, GA I/4, 44-45/FSW III, 242)

13. H. RICKERT, "Die philosophischen Grundlagen von Fichtes Sozialismus", Logos. Zeitschrift für systematische Philosophie, Heft 11, (1922/1923), pp. 149-180. Aquí: p. 179.

14. Este elemento impide hablar en Fichte de un socialismo colectivista, a pesar de su propuesta de una economía planificada centralmente. (A. RENAUT, Le Système de Droit. Philosophie et droit dans la pensée de Fichte, pp. 437-438)

15. Fichte apunta que el robo a un amigo o familiar en su domicilio suele ser considerado por la opinión pública como mucho más grave que el asalto en la calle, porque supone el abuso de la confianza y la buena fe de la víctima. Lo mismo constata respecto de la ofensa a un invitado por parte de su anfitrión y viceversa. (GNR §19, GA I/4, 45-46/FSW, III, 243)

16. Al respecto véase Z. BATSCHA, Gesellschaft und Staat in der politischen Philosophie Fichtes, Frankfurt a. M.: Europäische Verlagsanstalt, 1970, pp. 167-168.

\section{RESÚMENES}

In this paper I try to show that Fichte's theory of natural right does not consist in a form of collectivism, because individual freedom plays a fundamental role in the determination of some points of Fichte's natural right, such as his conception of money and inheritance as well as his observations about the privacy of the domestic realm.

ÍNDICE

Keywords: freedom, individual, theory of state

\section{AUTOR}

HÉCTOR ARRESE IGOR

La Plata 